\title{
Association between the inception of a SAFE KIDS Coalition and changes in pediatric unintentional injury rates
}

\author{
R F Tamburro, R I Shorr, A J Bush, S B Kritchevsky, G L Stidham, S A Helms
}

Injury Prevention 2002;8:242-245

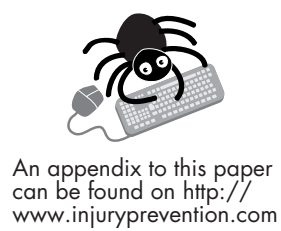

See end of article for authors' affiliations

Correspondence to Dr Robert F Tamburro, Division of Critical Care Medicine, St Jude Children's Research Hospital, 332 North Lauderdale, Memphis, TN 38105, USA;

robert.tamburro@stjude.org
Objective: To assess the relationship between the implementation of a SAFE KIDS Coalition and pediatric unintentional injury rates.

Setting: Shelby County, Tennessee.

Design: Retrospective observational analysis.

Patients: County residents nine years of age or younger presenting to the children's medical center, its emergency department, or its outpatient clinics from 1990-97.

Intervention: Implementation of a SAFE KIDS Coalition.

Main outcome measures: Rates of unintentional injuries targeted by the SAFE KIDS Coalition that resulted in hospitalization or in death. Rates of motor vehicle occupant injuries that resulted in hospitalization or in death. Rates of non-targeted unintentional injuries, namely injuries secondary to animals and by exposure to toxic plants. Rates of severe injuries (defined as those targeted injuries that required hospitalization or resulted in death), and specifically, severe motor vehicle occupant injuries were compared before and after the inception of the coalition using Poisson regression analysis.

Results: The relative risk of targeted severe injury rates decreased after implementation of the coalition even after controlling for changes in hospital admission rates. Specifically, severe motor vehicle occupant injury rates decreased $30 \%$ (relative risk $0.70 ; 95 \%$ confidence interval 0.54 to 0.89 ) after initiation of the coalition.

Conclusions: The implementation of a SAFE KIDS Coalition was associated with a decrease in severe targeted injuries, most notably, severe motor vehicle occupant injuries. Although causality cannot be determined, these data suggest that the presence of a coalition may be associated with decreased severe unintentional injury rates.
U nintentional injury remains the leading cause of death and disability in children in the United States. ${ }^{1-4}$ In response to this problem, the National SAFE KIDS Campaign was initiated by the National Children's Medical Center in 1987. It was the first nationwide program developed to address the problem of unintentional injury in children. ${ }^{56}$ The campaign has enlisted the support of corporations, government agencies, the health and safety community, and an ever-growing network of over 300 local and state coalitions, to reduce the incidence of unintentional injury. Under the sponsorship of a local organization, these coalitions develop injury prevention strategies based on identified risks and local resources, conduct public outreach and awareness campaigns, create task forces, conduct self assessment, and work to make injury prevention a public policy priority. A general calendar of activities conducted by the Mid-South SAFE KIDS Coalition is included in the Appendix (see www.injuryprevention.com).

Despite its widespread implementation, however, few studies have evaluated the relationship between SAFE KIDS and changes in injury rates. Other than national data, only the Harlem Hospital Injury Prevention Program, the lead organization for the Healthy Neighbors/SAFE KIDS Coalition, has published results of such an evaluation. ${ }^{6-8}$ Although they demonstrated a decrease in the incidence of injury among school aged children, their assessment was not focused specifically on the SAFE KIDS Coalition as they included other prevention efforts and evaluated additional injuries not targeted by the SAFE KIDS Campaign. ${ }^{78}$ Therefore, the purpose of the current study is to specifically assess the relationship between the implementation of a local SAFE KIDS Coalition and changes in pediatric unintentional injury rates.

\section{METHODS}

Study setting and population

Our study initially included all injuries occurring to Shelby County residents 9 years of age or younger presenting to the Le Bonheur Children's Medical Center, its emergency department, or any of its outpatient clinics from the years 1990-97. Injuries were identified using E code diagnoses from the hospital's billing database. Le Bonheur Children's Medical Center, which is the pediatric teaching facility of the University of Tennessee Health Science Center, is located in Shelby County. It is the only children's hospital in West Tennessee, providing care to more than 100000 children and adolescents annually, and during the study period, maintained the only pediatric emergency department in the region. Shelby County is located in the extreme southwest corner of the state of Tennessee and has Memphis as its largest city. It is the 43rd most populated county in the United States. ${ }^{9}$

We limited our analyses to injuries occurring in children less than or equal to 9 years of age. We did this because injured children 10 years of age and older are triaged to the adult trauma center (located across the street) when the children's medical center does not have available bed space due to high patient census. Thus, fluctuations in the number of injuries in the data base for children 10 years and older may reflect more variations in the need for diversion to the adult trauma center rather than true changes in the number of injuries.

For analysis, injuries were grouped into one of three age categories based on the age of the patient at the time of the injury ( $<1$ year, $1-4$ years, or $5-9$ years). This age grouping was chosen because it is the standard format of presenting injury data per the National Center for Injury Prevention and 
Control (Centers for Disease Control and Prevention). ${ }^{10}$ Population data were available based on these age groupings providing a denominator for analysis. ${ }^{11}$

\section{Injuries}

Targeted injuries, defined as those injuries actively addressed by the Mid-South SAFE KIDS Coalition, were identified and grouped into one of nine categories based on E code diagnoses from the International Classification of Diseases, ninth revision, using criteria obtained from the National SAFE KIDS Campaign. ${ }^{12}$ The National Center for Injury Prevention and Control has recommended similar groupings. ${ }^{10}$ These categories included:

(A) Motor vehicle occupant injury (E810.0-E819.9).

(B) Bicycle injury (E800.3, E801.3, E802.3, E803.3, E804.3, E805.3, E806.3, E807.3, E820.6, E821.6, E822.6, E823.6, E824.6, E825.6, E826.1, E826.9, E827.1, E828.1, E829.1).

(C) Burn injury (E890.0-E899.9).

(D) Drownings (E830.0-E830.9, E832.0-E832.9, E910.0-E910.9).

(E) Falls (E880.0-E886.9, E888.0-E888.9).

(F) Unintentional firearm injury (E922.0-E922.9).

(G) Suffocations (E911.0-E913.9).

(H) Pedestrian injury (E800.2, E801.2, E802.2, E803.2, E804.2, E805.2, E806.2, E807.2, E820.7, E821.7, E822.7, E823.7, E824.7, E825.7, E826.0, E827.0, E828.0, E829.0).

(I) Poisonings (E850.0-E869.9).

Bicycle and pedestrian injuries were subsequently condensed into one combined category. These categories were grouped because similar types of injuries were coded as E826.1 (bicycle) during the first six years of the study and as E826.0 (pedestrian) during the last two years. Additionally, injuries caused by animals and by exposure to toxic plants (E codes 905-906.9) were grouped into a 10th category labeled "animal and plant injuries". The Mid-South SAFE KIDS Coalition did not target these unintentional injuries. All other E code diagnoses were grouped into a category named "other" and were excluded from further analysis.

We limited our analysis to only severe injuries defined as those that required hospitalization or resulted in death. ${ }^{13-15}$ Only severe injuries were used in analysis for several reasons. First, many of the SAFE KIDS interventions are designed to minimize the severity of the injury rather than to eliminate the event altogether, in other words, secondary rather than primary prevention. For example, a restrained infant in a severe automobile collision or a helmeted bicyclist struck by a car may survive and not require hospitalization, but are likely to be evaluated in a medical facility. Consequently, these injuries would contribute to the overall injury rate, but not to the severe injury rate. Second, the need for hospitalization likely reduces referral bias. It is obvious that injured children receive medical care in a number of settings; however, when hospitalization is required, it is most likely that they will be admitted to the children's medical center. Third, severe injuries represent the most significant impact of unintentional injury in terms of morbidity, mortality, and cost.

Overall rates of severe injury were determined for the eight targeted categories for each calendar year using the number of injuries as the numerator and the annual age group, gender, and race specific population estimates for the base county as the denominator. The population estimates were obtained from the Tennessee Department of Health. ${ }^{.1}$ Severe injury rates within each targeted category were also determined for each age group for each calendar year.

\section{Statistical analyses}

Univariate statistics were performed using $\chi^{2}$ analysis. Multivariable analysis was performed with Poisson regression. A model was fit using a backwards deletion approach by first including all variables and all two way interactions. Only two way interactions were included for simplicity. Though Greenland has suggested that a change in parameter approach to model development may be superior to such a conventional approach, he also emphasized that significance testing is useful in evaluating interaction terms. ${ }^{16}$ Based on the overall descriptive data, it was anticipated that interaction terms would be important in this model, and thus, the decision was made to use a backwards deletion approach. Interaction terms that did not involve the variable for coalition era were retained only if they produced an important change in the estimated exposure effect. ${ }^{16}$ Two dependent variables were analyzed separately: severe targeted injuries and severe motor vehicle occupant injuries. Severe motor vehicle occupant injuries were analyzed since they were the single most targeted injury of the coalition. The models included a variable to define the eras before versus after the inception of the coalition. Since the coalition was not initiated until the last quarter of 1992 (year $3)$, years 1, 2, and $3(1990-92)$ were categorized as pre-coalition and years 4, 5, 6, 7, and 8 (1993-97) as post-coalition. Other variables included age group, race, and gender. To control for a potential secular trend related to a lower likelihood of hospital admission, we included annual Le Bonheur Children's Medical Center inpatient discharge counts in the models. The model for severe motor vehicle occupant injuries did not include the variables for age group and gender because the reduction in numbers associated with this focused assessment permitted no more than three variables in the model. Univariate analysis suggested that race would be the most important variable, in addition to annual inpatient discharge counts and the variable defining coalition eras.

Relative risks (RR) of injury before and after the inception of the coalition were determined for each model by exponentiating the estimated $\beta$ coefficient for coalition effect; $95 \%$ confidence intervals (CI) were determined using standard formulas. ${ }^{17}$ All statistical analyses were performed using the SAS 7.0 statistical software program (SAS Institute Inc, Cary, North Carolina). The study was approved by the University Institutional Review Board and the need for informed consent was waived.

\section{RESULTS}

A total of 27776 injuries targeted by the SAFE KIDS Coalition occurring in children less than 10 were initially identified between the years 1990-97, of which 2923 (11\%) resulted in either death or hospitalization. The rate of severe targeted injuries progressively decreased from its maximum of 3.5 per 1000 during the first two years of the study to its nadir of 2.0 per 1000 attained during year 7 of the study (1996). Moreover, rates of animal and plant injuries, unintentional injuries not targeted by the coalition increased from a nadir of 1.4 per 1000 person during the first year of the study (1990) to a maximum of 2.5 per 1000 person obtained during the last year of the study (fig 1). Figure 2 plots the severe injury rates per year in the study county for the categories of injury targeted by the coalition as well as the national mortality rates for unintentional injury for the same age group. ${ }^{4}$

The decrease in severe injury rates for targeted conditions (RR $0.77 ; 95 \%$ CI 0.66 to 0.90 ) detected after the inception of the coalition was noted even after controlling demographic and secular trends in decreasing hospital admission rates. However, there was a significant gender-coalition interaction, and thus, separate relative risks were determined for males and females. For males, a significant decrease in the relative risk of targeted unintentional injury was noted (RR 0.77; 95\% CI 0.66 to 0.90 ). For females, the impact was tempered by the gender-coalition interaction yielding a statistically insignificant decrease 0.93 (95\% CI 0.79 to 1.09 ).

Table 1 depicts the severe injury rate for the specific categories of injury. For children less than 1 year, falls were the leading cause of severe injury followed distantly by motor vehicle occupant injuries, poisonings, and suffocations. Though the numbers are small, there does appear to be a decrease in the 


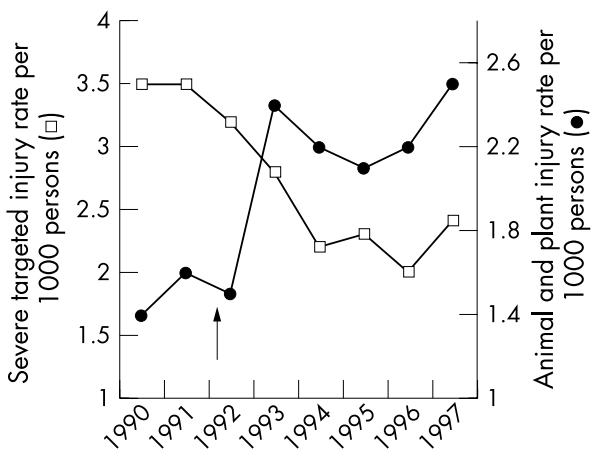

Figure 1 A comparison of severe targeted unintentional injury rates with rates of injury secondary to animals and exposure to toxic plants within the study county in children 0-9 years. The arrow depicts the inception of the coalition.

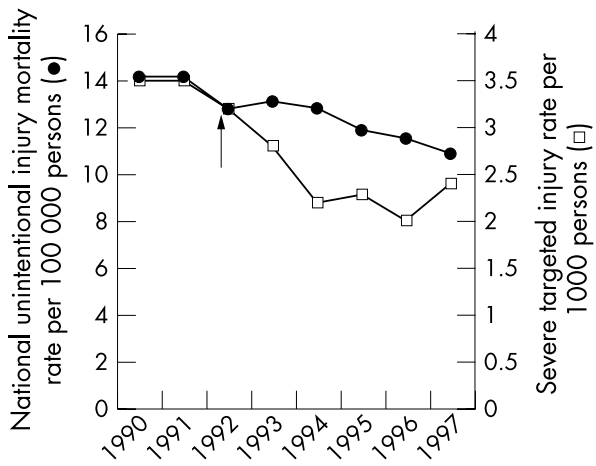

Figure 2 A comparison of severe targeted unintentional injury rates in the study county with national mortality rates for unintentional injuries in children 0-9 years during the same time period. The arrow depicts the inception of the coalition. ${ }^{4}$

incidence of suffocations in the post-coalition era. Poisonings were the leading cause of severe injuries for the $1-4$ age group, and motor vehicle occupant injuries for the 5-9 age group. Of note, the rate of severe motor vehicle occupant injuries appears to have decreased in the post-coalition period. This was borne out in analysis as the post-coalition $\beta$ coefficient estimated the relative risk of severe motor vehicle occupant injury after the inception of the coalition to be 0.70 (95\% CI 0.54 to 0.89 ) of the pre-coalition era.

\section{DISCUSSION}

The implementation of a local SAFE KIDS Coalition was temporally associated with a decrease in the severe injury rate among targeted conditions. This association was noted while controlling for secular trends in hospital admissions. Specifically, the coalition was associated with a 30\% decrease in the relative risk of severe motor vehicle occupant injuries.

Though certainly no case for causality can be offered from this observational analysis of secular trends, the findings do suggest an effect that is at least consistent with that of the coalition. First, there was an association between the inception of the coalition and a decrease in severe injuries. Severe unintentional injury rates in Shelby County mirrored national unintentional injury mortality during the precoalition era for children less than 10 years. However, after the inception of the coalition, the decrease in Shelby County rates was seemingly more marked than that of the national data (fig 2). ${ }^{4}$ Additionally, the finding of decreased severe motor vehicle occupant injury rates is also consistent with an effect of the coalition since this was the most actively addressed category of injury. This finding is particularly noteworthy since available national data suggest that mortality from childhood motor vehicle occupant injury remained essentially unchanged during that time period. ${ }^{6}{ }^{18} 19$ Moreover, the progressive increase in the rate of "non-targeted" animal and plant associated injuries also suggests an effect compatible with that of the coalition. Though the increase in these animal and plant associated injuries may be attributed to many factors, the difference in the trend of these injuries and that of the targeted unintentional injuries is at least consistent with an effect of the coalition.

This study has several limitations. First, this study is limited by the very nature of its design. This observational study can

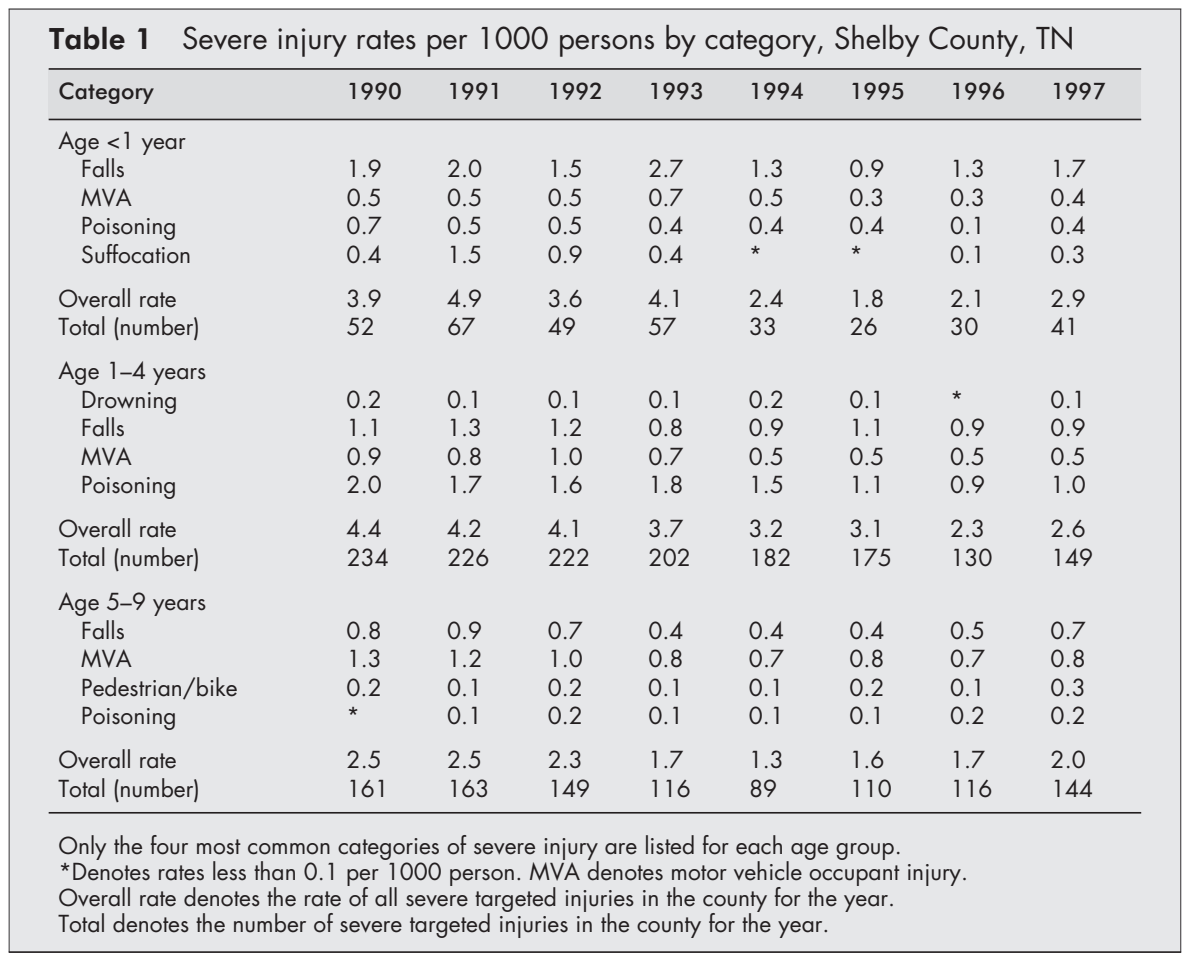


at most detect temporal associations between injury rates and the inception of the coalition. Ideally, a comparison with a control county without a coalition matched on socioeconomic, population, and geographic parameters over the same time period would strengthen the assessment. However, with coalitions currently in place in over 300 cities and all 50 states, it is essentially impossible to identify a comparable county without a SAFE KIDS program that has meaningful data for the time period. A comparison with injury rates among non-targeted age groups within the study county would also improve the overall evaluation of the coalition. Davidson and the group from Harlem used this type of comparison as well as a control community in their analysis. ${ }^{8}$ Unfortunately, our access to data for other age groups was limited, and thus, we could not conduct this analysis. Consequently, our comparison was limited to non-targeted unintentional injuries (that is, "animal and plant injuries") among children of the same age group within the county and to national mortality data.

Additionally, confounders other than secular trends in admission rates (that is, changes in health care delivery, improvements in the safety features of toys, automobiles, and bedding equipment, the expansion of the internet, etc) may have influenced unintentional injury rates during this time period and could not be controlled. The implementation of TennCare is a notable example. TennCare is a statewide health care system reform plan that was developed to control Medicaid costs and to extend health insurance coverage to most Tennesseans. ${ }^{20}$ This program, however, was not even initiated until 1994, and a decrease in the targeted injury rate was already noted by 1993 suggesting an effect independent of TennCare. Also, Shelby County underwent considerable growth during this time period with expansion in many housing developments, roadways, and businesses. Certainly this growth could have influenced injury rates. Additionally, a mandatory bicycle helmet law was enacted in 1994 on a statewide basis. Obviously, this may have influenced bicycle related injuries. These represent but a few of the potential confounding activities.

Another limitation of this study is that injury identification depended on appropriate coding by the medical records department. E codes had been documented at the medical center for at least two years before the onset of the study, and thus, the coders would seemingly be facile with the system. Moreover, if coding improved over the course of the study, more injuries should have been identified, thus making it more difficult to demonstrate a positive effect of the coalition. Finally, the collection of data from a single medical center system represents another weakness as it is certain that children received care for unintentional injury at other medical facilities. However, by focusing analysis on only severe injuries, it is hoped that this limitation was minimized as it is most likely that injured children were admitted to the children's medical center when hospitalization was required.

Despite these acknowledged limitations, our study suggests that there was a temporal relationship between the implementation of a SAFE KIDS Coalition and decreases in targeted severe injury rates in children in Shelby County, Tennessee. Severe motor vehicle occupant injuries were targeted extensively by the local SAFE KIDS initiative, and fell significantly after implementation. In sum, the SAFE KIDS Coalition appears to have been associated with a beneficial effect in this analysis, but further studies are needed to establish whether this approach to injury prevention in children is uniformly effective.

\section{ACKNOWLEDGEMENTS}

Grant support: this study was funded in part by the 21st Century Scholars Program, LHS Inc. The study was approved by the University of Tennessee Health Science Center Institutional Review Board and the need for informed consent was waived.

\section{Key points}

- The implementation of a local SAFE KIDS Coalition was temporally associated with a decrease in targeted injuries.

- Non-targeted unintentional injuries increased during the study time period.

- Severe motor vehicle occupant injuries were targeted extensively by the local SAFE KIDS initiative, and fell significantly after implementation.

- This occurred despite national data suggesting mortality rates from such injuries remained essentially unchanged during this time.

- The SAFE KIDS Coalition appears to have been associated with a beneficial effect, but further studies are needed to establish the effectiveness of this approach to injury prevention.

\section{Authors' affiliations}

R F Tamburro, Division of Critical Care Medicine, St Jude Children's Research Hospital, Department of Pediatrics, Le Bonheur Children's

Medical Center, and Department of Preventive Medicine, University of

Tennessee Health Science Center, Memphis, Tennessee

R I Shorr, Department of Preventive Medicine, University of Tennessee Health Science Center and Department of Medical Education, Methodist Healthcare, Memphis, Tennessee

A J Bush, S B Kritchevsky, Department of Preventive Medicine,

University of Tennessee Health Science Center, Memphis, Tennessee

G L Stidham, Department of Pediatrics, Le Bonheur Children's Medical

Center, Memphis, Tennessee

S A Helms, Injury Prevention, Le Bonheur Children's Medical Center and Mid-South SAFE KIDS Coalition, Memphis, Tennessee

\section{REFERENCES}

1 Centers for Disease Control and Prevention, Division of Injury Control, Centers for Environmental Health and Injury Control. Childhood injuries in the United States. Am J Dis Child 1990;144:627-46.

2 Guyer B, Hoyert DL, Martin JA, et al. Annual summary of vital statistics. Pediatrics 1999;104:1229-46.

3 Brenner RA, Overpeck MD, Trumble AC, et al. Deaths attributable to injuries in infants, United States, 1983-1991. Pediatrics 1999; 103:968-74.

4 Centers for Disease Control. Web-based injury statistics query and reporting system (WISQARS) (http://www.cdc.gov/ncipc/wisqars), May 2002.

5 Mickalide A. The National SAFE KIDS Campaign. Inj Prev 1995;1:119-21.

6 O'Donnell GW, Mickalide AD. SAFE KIDS at home, at play, and on the way: a report to the nation on unintentional childhood injury. Washington, DC: National SAFE KIDS Campaign, 1998.

7 Laraque $D$, Barlow $B$, Durkin $M$, et al. Injury prevention in an urban setting: challenges and successes. Bull N Y Acad Med 1995;72:16-30.

8 Davidson LL, Durkin MS, Kuhn L, et al. The impact of the Safe Kids/Healthy Neighborhoods Injury Prevention Program in Harlem, 1988 through 1991. Am J Public Health 1994;84:580-6.

9 Shelby County Web Page (http://www.co.shelby.tn.us/home/ facts. htm).

10 Centers for Disease Control and Prevention. Recommended framework for presenting injury mortality data. MMWR Morb Mortal Wkly Rep 1997;46:1-29

11 Tennessee Department of Health. SPOT Statistical Profiling of Tennessee (http://web.utk.edu/ chrg/stdtnpop.htm).

12 World Health Organization. International classification of diseases. 9th Revision. Geneva, Switzerland: World Health Organization, 1980: El-27.

13 Davidson LL, Durkin MS, O'Connor PO, et al. The epidemiology of severe injuries to children in northern Manhattan: methods and incidence rates. Paediatr Perinatal Epidemiol 1992;6:153-65.

14 Hu X, Wesson DE. Fatal and non-fatal childhood injuries in Metropolitan Toronto, 1986-1991. Can J Public Health 1994;85:269-73.

15 Lapidus G, Lerer T, Zavoski R, et al. Childhood injuries in Connecticut. Conn Med 1998;62:323-31.

16 Greenland S. Modeling and variable selection in epidemiologic analysis. Am J Public Health 1989;79:340-9

17 Kleinbaum DG, Kupper LL, Morgenstern H. Modeling: theoretical considerations. Epidemiologic research. Belmont, California: Lifetime Learning Publications, 1982: 432-3.

18 Durbin DR. Preventing motor vehicle injuries. Curr Opin Pediatr $1999 ; 11: 583-8$

19 Motor-vehicle occupant fatalities and restraint use among children aged 4-8 years United States, 1994-1998. MMWR Morb Mortal Wkly Rep 2000;49: 135-7.

20 Mirvis DM, Chang CF, Hall CJ, et al. TennCare-health system reform for Tennessee. JAMA 1995;274:1235-41. 